\title{
ESTUDO DA INFLUÊNCIA DA SÍLICA E DO NEGRO DE FUMO NAS PROPRIEDADES MECÂNICAS DOS COMPOSTOS DE BORRACHA APLICADOS EM PNEUS*
}

Karen Leão dos Santos ${ }^{1}$ Marcelo Gonçalves ${ }^{2}$

\section{Resumo}

A borracha sintética (SBR) é amplamente utilizada em indústrias de pneus combinada a cargas de reforço para melhoria das propriedades do elastômero puro. Atualmente, as cargas que apresentam melhor capacidade de reforço em elastômeros são a sílica e o negro de fumo. O presente estudo buscou avaliar o desempenho de compostos de borracha sobre as características de resistência ao rolamento, tração em piso molhado e resistência à abrasão, em função das quantidades de sílica e de negro de fumo. Foram realizados ensaios mecânicos e dinâmico-mecânicos, e, a partir dos resultados obtidos, concluiu-se que a utilização das diferentes cargas de reforço no composto de borracha está associada ao tipo de uso a que se destina o pneu.

Palavras-chave: Elastômeros; Sílica; Negro de fumo; Cargas de reforço.

\section{THE INFLUENCE OF CARBON BLACK AND SILICA ON THE MECHANICAL PROPERTIES OF ELASTORMER BLENDS APPLIED TO TYRES}

\begin{abstract}
Synthetic rubbers (SBR) are widely used in tyre manufacturing as a filler agent in order to improve the elastomers properties. Silica and carbon black are the filler agents that have a greater yield to enhance the mechanical properties of rubber. The wet grip, the rolling resistance and the abrasion resistance for different amounts of silica and carbon black in the rubber compounds were studied. Results have shown that the optimized choice of each filler agent is associated to the specific use of the tyre during its life-time.
\end{abstract}

Keywords: Elastomer; Silica; Carbon black; Filler.

Engenheira de Produção, ESEG - Escola Superior de Engenharia e Gestão, São Paulo, Brasil.

2 Engenheiro Metalurgista, M.E, PhD, ESEG - Escola Superior de Engenharia e Gestão e Alpina Consultoria, São Paulo, Brasil. 


\section{INTRODUÇÃO}

Elastômeros são materiais poliméricos que apresentam elevada elasticidade, e têm uma grande gama de aplicações, dentre elas, pneus de veículos automotores [1, 2]. Em suas composições básicas, os elastômeros apresentam propriedades mecânicas limitadas, havendo a necessidade de adição de cargas de reforço para torná-los adequados e resistentes para aplicação em pneus [3].

Das cargas particuladas de reforço utilizadas em compostos de borracha nas indústrias de pneus, destacam-se a sílica e o negro de fumo, sendo que a sílica se tornou relevante apenas recentemente devido à sua capacidade de proporcionar características únicas em comparação ao negro de fumo [2, 4].

A sílica utilizada como carga de reforço em bandas de rodagem melhora simultaneamente as propriedades de aderência do pneu ao piso molhado e de resistência ao rolamento. Essas duas características (aderência ao piso molhado e resistência ao rolamento), conjugadas à resistência à abrasão, formam o "triângulo mágico", são as características mais importantes a serem consideradas para o desempenho ótimo dos pneus $[1,2,5]$.

O uso de cargas de reforço nos elastômeros melhora o desempenho dos pneus, principalmente em relação à resistência ao rasgamento, à tração e à abrasão. Embora essas propriedades sejam importantes para caracterizar os compostos, elas devem ser completadas com testes dinâmico-mecânicos, que medem a deformação do elastômero sob aplicação de forças cíclicas [5]. É considerado que, durante o uso de um pneu, as tensões aplicadas e a deformações resultantes variam de forma senoidal com o tempo, e a quantificação de ambas permite obter o módulo elástico e - módulo viscoso. O módulo elástico, também chamado de módulo de armazenamento (E'), mede a quantidade de energia elástica armazenada pela borracha, e, o módulo viscoso, também denominado módulo de perda (E"), representa a parte da energia dissipada de forma secundária, como a perda na forma de calor, definida como a perda por histerese [7]. Essas propriedades estão intrinsecamente relacionadas com a adição de cargas no composto, o que acarreta mudanças nos dois módulos, bem como a razão entre eles (E'/E'), razão esta denominada como fator de amortecimento, tanঠ $[5,7]$.

Nos testes dinâmico-mecânicos, normalmente obtém-se a chamada curva de tanঠ que possibilita a avaliação do comportamento viscoelástico do composto. A análise da curva em faixas de temperaturas específicas permite fazer inferências sobre as propriedades do "triângulo mágico" [6,7]. Do ponto de vista das propriedades viscoelásticas, para que o pneu apresente baixa resistência ao rolamento, 0 composto ideal deve apresentar um baixo valor de tan $\delta$ entre $30-70^{\circ} \mathrm{C}$. Por outro lado, para se obter elevada aderência à pista molhada, o composto deve apresentar um alto valor de tanס, ou seja, alta histerese, a temperaturas mais baixas [6].

Neste contexto, o objetivo deste trabalho foi desenvolver um estudo comparativo a fim de se estudar os efeitos de diferentes concentrações de cargas de reforço nos compostos de SBR, sobre as propriedades denominadas como "triângulo mágico", a saber, a resistência ao rolamento, resistência à abrasão e aderência ao piso molhado [3]. Assim, faz-se necessário estudar a influência das propriedades desses dois reforços sobre o desempenho do pneu, tendo-se em vista a crescente preocupação com a durabilidade dos pneus e a economia de combustível [6].

O presente estudo objetiva, portanto, ser uma contribuição para o aprofundamento do conhecimento sobre os efeitos desses dois tipos de cargas usadas em 
compostos de borrachas aplicadas em pneus, abordando o problema de um ponto de vista tecnológico de engenharia de materiais.

\section{MATERIAIS E MÉTODOS}

Os compostos foram desenvolvidos e caracterizados quanto às propriedades dinâmico-mecânicas no laboratório da empresa Pirelli Pneus Ltda., em Santo André. Para realização do estudo experimental foram utilizados compostos de borracha definidos na Tabela 1 e 2.

Tabela 1. Formulação dos compostos de elastômero utilizados na avaliação das propriedades mecânicas e dinâmico-mecânicas

\begin{tabular}{ccc}
\hline INGREDIENTE & DESCRIÇÃO & PHR $^{*}$ \\
\hline SBR 1502 & Copolímero aleatório estireno-butadieno (23,5\% estireno, 13\% & 100 \\
TESPT & vinil) Tg -50 ${ }^{\circ}$ (Borracha) & Variável \\
Negro de Fumo & begro de fumo N234 (Carga reforçante) & Variável \\
Sílica & Dióxido de silício (Carga reforçante) & Variável \\
Ácido Esteárico & Ácido Esteárico (Ativador da vulcanização) & 1,5 \\
ZnO & Óxido de Zinco (Ativador da vulcanização) & 1 \\
6PPD & $\mathrm{N}$-(1,3-dimetilbutil)-N'-fenil-p-fenilenoamina & 2 \\
DPG & (Antioxidante/Antiozonante) & Variável \\
CBS & 1,3-difenilguanidina (Catalizador da silanização) & 2,4 \\
ZBDC & $\mathrm{N}$-ciclohexil-2-benzotiazol-2-sulfenamida (Acelerante) & 0,35 \\
Enxofre & dibutil ditiocarbamato de zinco (Acelerante) & 1 \\
\hline${ }^{*}$ PHR (Per Hundred Rubber), o termo significa partes de qualquer material não-borracha a cada cem partes de & borracha
\end{tabular}

Tabela 2. Nomenclatura das composições utilizadas em cada formulação

\begin{tabular}{ccccc}
\hline COMPOSTO & NF (PHR) & SÍLICA (PHR) & TESPT (PHR) & DPG (PHR) \\
\hline $00-60$ & 0 & 60 & 4,20 & 1 \\
$15-45$ & 15 & 45 & 3,15 & 0,76 \\
$30-30$ & 30 & 30 & 2,10 & 0,50 \\
$45-15$ & 45 & 15 & 1,05 & 0,25 \\
$60-00$ & 60 & 0 & 0 & 0 \\
\hline
\end{tabular}

Após a definição dos ingredientes, estes foram colocados em um misturador fechado tipo Banbury de 4 pás da marca Copé com volume de 1,5 L. Ao final do processo de mistura de cada uma das fases (definidas na Tabela 3), os compostos foram colocados em um misturador de cilindros abertos para homogeneização e obtenção de mantas, sendo em seguida resfriados em um banho de água na temperatura ambiente "visando evitar a reação de vulcanização prematura do composto pelo aquecimento gerado no processo de mistura" [8]. Para cada fase, além dos ingredientes descritos na Tabela 3, a manta confeccionada na fase de mistura anterior também foi introduzida no misturador fechado (Banbury). 
Tabela 3. Ciclos de mistura utilizado no desenvolvimento dos compostos

\begin{tabular}{|c|c|c|c|c|}
\hline FASE & INGREDIENTES & $\begin{array}{l}\text { TEMPO } \\
\text { (S) }\end{array}$ & $\begin{array}{l}\text { VELOCIDADE } \\
\text { (RPM) }\end{array}$ & $\begin{array}{c}\text { TEMPERATURA } \\
\left({ }^{\circ} \mathrm{C}\right)\end{array}$ \\
\hline 1ㅁ Fase & $\begin{array}{l}\text { SSBR + Sílica + Silano + } \\
\text { negro de fumo }\end{array}$ & 195 & 70 & 145 \\
\hline $2^{\mathrm{a}}$ Fase & $\begin{array}{c}\text { ZnO + ácido esteárico + } \\
\text { 6PPD }\end{array}$ & 175 & 70 & 140 \\
\hline $3^{a}$ Fase & $\begin{array}{c}\mathrm{DPG}+\mathrm{ZBDC}+\text { enxofre + } \\
\mathrm{CBS}\end{array}$ & 90 & 70 & 100 \\
\hline
\end{tabular}

O processo foi repetido para as cinco formulações, variando-se as quantidades de sílica e negro de fumo (e os aditivos correspondentes TESPT e DPG), como apresentado na Tabela 2. Terminada a última fase de mistura dos compostos, as mantas passaram por um processo de descanso de aproximadamente 16 horas e foram posteriormente direcionadas para preparação das amostras.

$\mathrm{Na}$ preparação, as mantas foram cortadas, pesadas e colocadas em moldes préaquecidos a fim de serem inseridas na prensa vulcanizadora para moldagem do corpo de prova durante o processo de vulcanização. Foram colocados na prensa vulcanizadora com uma pressão de $50 \mathrm{kgf} / \mathrm{cm}^{2}$ à temperatura de $170^{\circ} \mathrm{C}$ por 10 minutos. O processo foi o mesmo para todos os compostos. Depois da desmoldagem dos corpos de prova, estes foram introduzidos em um banho de água a temperatura ambiente visando interromper a vulcanização. Os corpos de prova vulcanizados passaram por um processo de descanso de aproximadamente 30 horas e foram direcionados aos ensaios.

\subsection{Ensaio de resistência à abrasão}

O ensaio da resistência à abrasão, determinado conforme a norma DIN 53516, foi realizado no abrasímetro Maqtest, constituído por um rolete $(150 \pm 0,2 \mathrm{~mm}$ de diâmetro e $50 \mathrm{~cm}$ de comprimento), revestido por uma lixa de abrasividade padronizada, que girava com velocidade de rotação de $40 \mathrm{rpm}$.

O corpo de prova cilíndrico do composto vulcanizado $(9,8 \mathrm{~mm}$ de altura e $16 \pm 0,1$ $\mathrm{mm}$ de diâmetro), submetido a uma força peso de $10 \mathrm{~N}$, foi deslocado sobre o rolete, atingindo a velocidade constante de $0,32 \mathrm{~m} / \mathrm{s}$ e percorrendo uma distância total de $40 \mathrm{~m}$. Ao final do percurso, o corpo é pesado novamente para o cálculo do volume perdido.

Para cada composto foram ensaiados quatro corpos de prova, obtendo-se o resultado médio de resistência à abrasão. Para o cálculo da resistência, considerava-se o volume $\left(\mathrm{mm}^{3}\right)$ do material perdido após o desgaste da lixa. A perda de volume $(\Delta V)$ foi calculada pela Equação 1:

$$
\Delta V=\frac{\Delta_{m} \times S_{0}}{\mu u \times S}
$$

Onde $\underline{\Delta}_{\mathrm{m}}$ é a perda de massa calculada pela diferença do peso da amostra antes e depois do ensaio, $\underline{S}_{0}$ é a constante da lixa utilizada $(200 \mathrm{mg})$, $\mu u$ é a densidade do composto (mg. $\left.\mathrm{mm}^{-3}\right)$ e $\underline{\mathrm{S}}$ a abrasividade da lixa $(0,190 \mathrm{mg} \cdot 40 \mathrm{~m})$. 


\subsection{Ensaio dinâmico mecânico: MTS}

A medição das propriedades dinâmico-mecânicas dos compostos foi realizada no equipamento MTS modelo 831 Elastomer System, em que os corpos de prova foram solicitados em regime dinâmico de compressão para obtenção dos módulos de perda (E”) e de armazenamento (E'), e do fator de amortecimento (tan $\delta$ ).

O corpo de prova em formato cilíndrico $(25 \mathrm{~mm}$ de comprimento e $18 \mathrm{~mm}$ de diâmetro), após a estabilização da temperatura na estufa, foi posicionado no atuador dinâmico do equipamento. Ao iniciar o teste, o corpo sofreu uma pré-deformação de $25 \%$ na amplitude, com uma carga de $3,5 \mathrm{~N}$ durante dois minutos. Em seguida, houve um pré-condicionamento mecânico, em que houve a aplicação da mesma força em 125 ciclos a 100Hz, com uma deformação de 5\% (amplitude de deformação equivalente a $10 \%$, para eliminação da memória viscoelástica do composto.

Após o pré-condicionamento mecânico da amostra, ainda com a pré-deformação aplicada, o corpo de prova foi submetido a uma deformação por compressão de $3,5 \%$ a $1 \mathrm{~Hz}, 10 \mathrm{~Hz}$ e $100 \mathrm{~Hz}$, à temperatura de $0^{\circ} \mathrm{C}$. $\mathrm{O}$ procedimento foi repetido em sua totalidade também para as temperaturas de 23 e $70^{\circ} \mathrm{C}$, utilizando-se as mesmas amostras.

O valor do módulo de armazenamento E', do módulo de perda E" e do fator de amortecimento $\tan \delta$ foram obtidos à temperatura de $0^{\circ} \mathrm{C}$ e $70^{\circ} \mathrm{C}$, à frequência de $10 \mathrm{~Hz}$.

Por meio dos resultados obtidos no MTS, foi possível analisar a resistência ao rolamento e aderência ao piso molhado dos compostos, correlacionando estas características com o coeficiente $\tan \delta$ a $70^{\circ} \mathrm{C}$ e $10 \mathrm{~Hz}$, e a $0^{\circ} \mathrm{C}$ e $10 \mathrm{~Hz}$, respectivamente.

\section{RESULTADOS E DISCUSSÃO}

\subsection{Ensaio de resistência a abrasão}

A partir dos resultados obtidos de resistência à abrasão, foi possível observar uma diminuição de desgaste por abrasão conforme o aumento da quantidade de negro de fumo no composto (Figura 1).

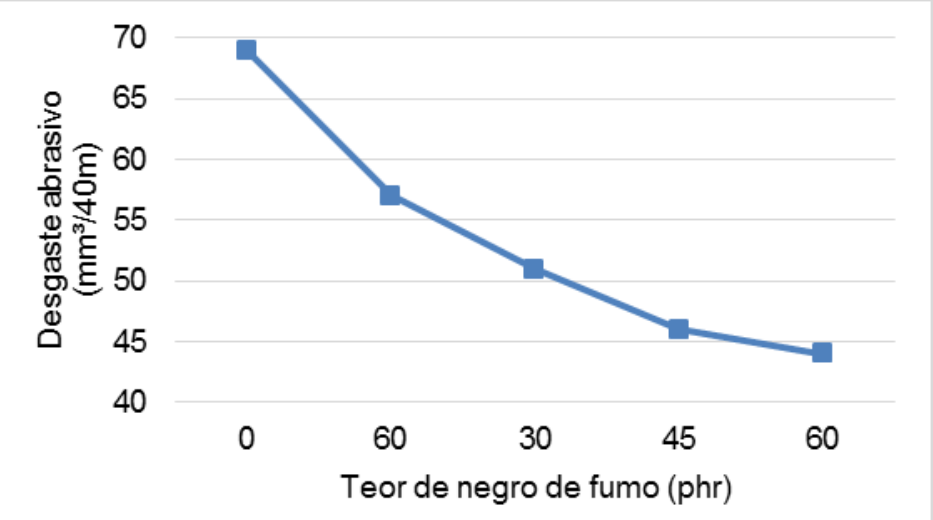

Figura 1. Desgaste abrasivo $\left(\mathrm{mm}^{3} \cdot 40 \mathrm{~m}^{-1}\right)$ em função da quantidade de negro de fumo (phr).

Este comportamento se dá pela diferente interação que existe entre a matriz polimérica e as cargas de reforço. No caso do negro de fumo, tanto a carga quanto a 
matriz são apolares e apresentam uma interação mais forte entre si, sem a necessidade de um agente de acoplamento. Esta interação entre a carga e a matriz ocorre em mais pontos para o negro de fumo do que para a sílica e faz com que o composto apresente maior resistência ao desgaste. No caso da sílica, o desgaste abrasivo é maior. Conforme aumenta-se o conteúdo dessa carga de reforço, o desgaste abrasivo também aumenta (Figura 2), devido a interação do polímero e da carga [2].

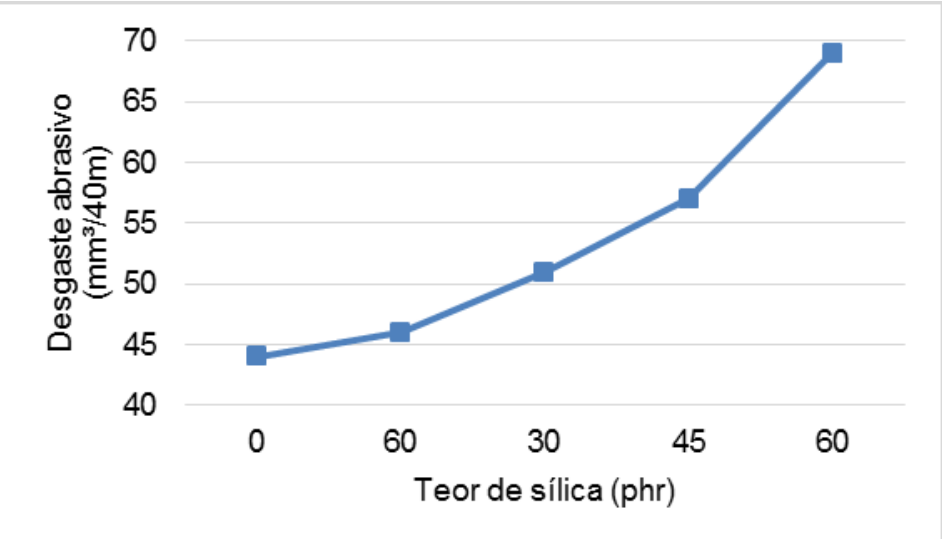

Figura 2. Desgaste abrasivo ( $\left.\mathrm{mm}^{3} / 40 \mathrm{~m}\right)$ em função do conteúdo de sílica

O rendimento quilométrico do pneu está intrinsecamente relacionado com o desgaste abrasivo: quanto maior a perda de volume, menor o rendimento quilométrico, portanto, menor a vida-útil do pneu. Desse modo, diante dos resultados aqui apresentados, percebe-se que quanto maior a concentração de sílica, maior o desgaste e menor o rendimento quilométrico, diminuindo-se também a vida-útil do pneu.

\subsection{Ensaio dinâmico-mecânico: MTS}

As respostas elástica ( $E^{\prime}$ ) e plástica (E”) do composto em $70^{\circ} \mathrm{C}$ auxiliam na predição da resistência ao rolamento do pneu, sendo que, quanto maior a resposta elástica (módulo de armazenamento) com relação a resposta plástica (módulo de perda), menor a histerese do composto e menor a resistência ao rolamento. No entanto, deve ser analisada a razão entre um e outro, ou seja, o coeficiente $\tan \delta$.

Observa-se nos resultados a $70^{\circ} \mathrm{C}$ e $10 \mathrm{~Hz}$, um aumento do tan $\delta$ relacionado ao aumento do conteúdo de negro de fumo (Figura 4).

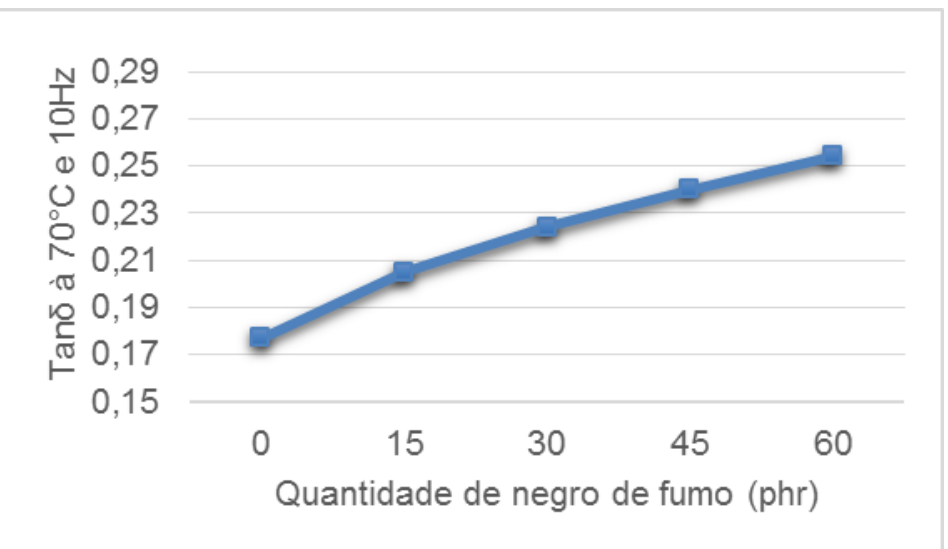

Figura 4. Comportamento do tanঠ a $70^{\circ} \mathrm{C}$ e $10 \mathrm{~Hz}$ em função da quantidade de negro de fumo. 
O negro de fumo gera maior histerese no composto, o que se deve ao fato de que o negro de fumo reduz a fração polimérica do composto e aumenta o atrito interno polímero-carga [10], ou seja, tem-se maior perda em forma de calor, o que consequentemente gera o aumento do fator de amortecimento (tan $\delta$ ), quando comparado a um composto com maior conteúdo de sílica (Figura 5).

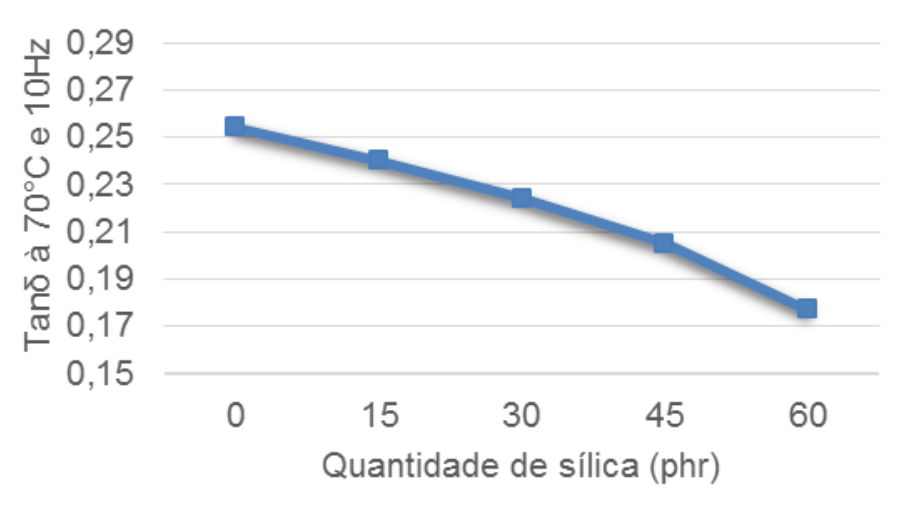

Figura 5. Comportamento do tanס a $70^{\circ} \mathrm{C}$ e $10 \mathrm{~Hz}$ em função da quantidade de sílica.

Como mencionado pela literatura, as cargas de origem mineral, como a sílica, utilizadas com o agente de acoplamento, tendem a apresentar resultados mais elevados de energia restituída (maior resposta E'), podendo isso estar relacionado com a melhor interação entre a sílica e o polímero [3].

Dessa forma, para utilização do composto em bandas de rodagem de baixa resistência ao rolamento, o composto 60-0 é o menos adequado (Figura 6), pois, por ter um alto fator de amortecimento, gera maior resistência ao rolamento do pneu e, consequentemente, aumenta o gasto de combustível.

O composto 0-60 é o mais indicado para aplicação às bandas de rodagem em relação à resistência ao rolamento. Por possuir o módulo de amortecimento menor, apresentará menor resistência ao rolamento.

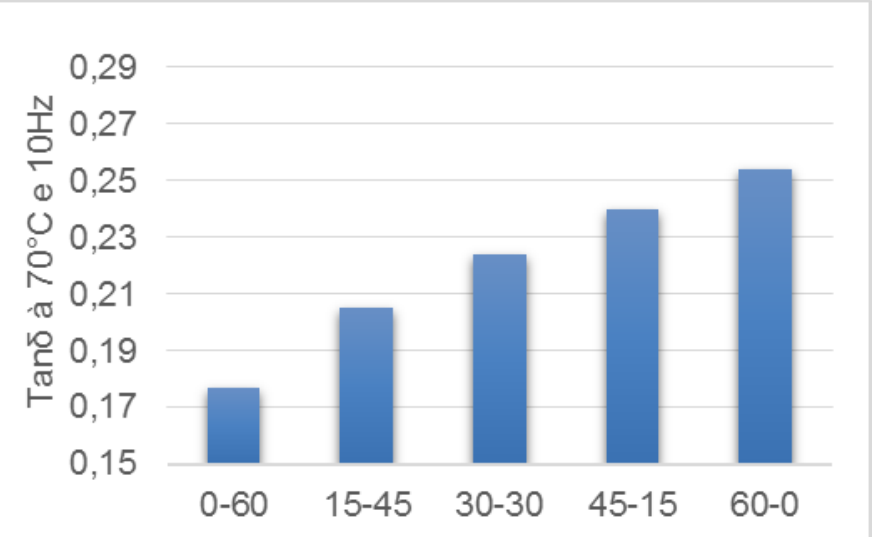

Figura 6. Resultados dos módulos de amortecimento (tanঠ) dos compostos

Com relação à aderência em piso molhado, quanto maior o valor do tanঠ a $0^{\circ} \mathrm{C} \mathrm{e}$ $10 \mathrm{~Hz}$, melhor o composto. Com o valor alto de tanঠ, obtém-se um composto mais plástico e rígido e, para desempenho do pneu no piso molhado, quanto menor o valor do E', melhor o desempenho no pneu. A baixas temperaturas é esperado que o pneu tenha alta histerese (maior componente plástica) para se moldar melhor às 
imperfeições do solo, garantindo boa dirigibilidade e frenagem do pneu, características de extrema importância relacionadas à segurança.

Para analisar os resultados dessa característica, obtidos a $0^{\circ} \mathrm{C}$ e $10 \mathrm{~Hz}$, foi utilizada a relação proposta por Medalia et al.[9] na qual consideram que a energia perdida a uma carga constante, para compostos de banda de rodagem, é proporcional a $E^{\prime \prime} /\left(E^{\prime}\right)^{2}$. Usando-se esta razão, o melhor desempenho em termos de aderência ao piso molhado resultou para a amostra 0-60 (Figura 7).

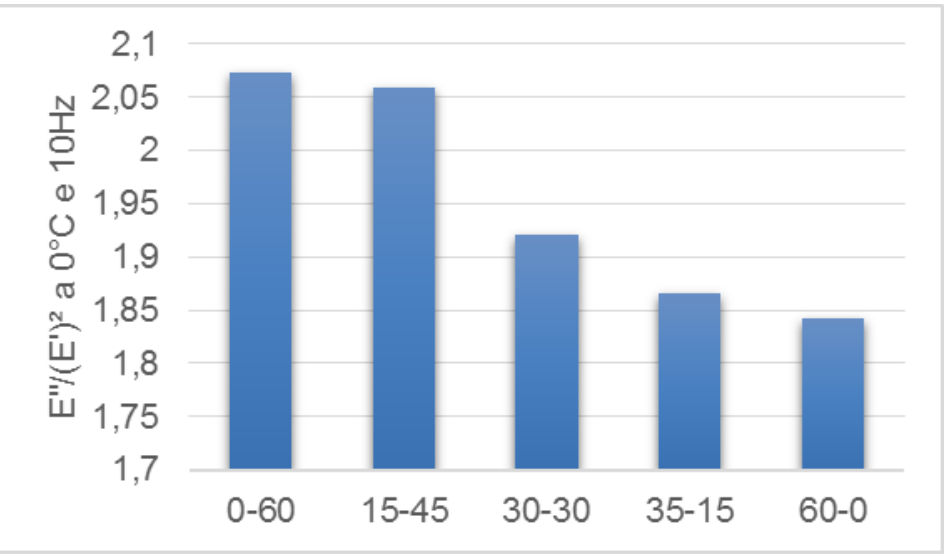

Figura 7. Resultados de $\mathrm{E}^{\prime \prime} /\left(\mathrm{E}^{\prime}\right)^{2}$ a $0^{\circ} \mathrm{C}$ e $10 \mathrm{~Hz}$

\subsection{Construção do "Triângulo Mágico"}

Considerando-se os resultados apresentados, é possível selecionar características para formação do triângulo mágico (Figura 8). $\mathrm{O}$ valor do tanঠ à $70^{\circ} \mathrm{C}$ e $10 \mathrm{~Hz}$, obtido no equipamento MTS, foi utilizado para representar a resistência ao rolamento. A relação $\mathrm{E}^{\prime \prime}\left(\mathrm{E}^{\prime}\right)^{2}$ nas condições de $0^{\circ} \mathrm{C}$ e $10 \mathrm{~Hz}$, também obtida no equipamento MTS, foi utilizada para o valor de aderência ao piso molhado. Por fim, a quantificação do desgaste abrasivo foi obtida a partir do ensaio de Abrasão DIN, no abrasímetro Maqtest. Tais dados estão apresentados de maneira consolidada no "triângulo mágico" da Figura 8, que representa a síntese em termos das características de desempenho desejáveis no pneu automotivo.

Ao analisar a Figura 8, para determinar-se o equilíbrio entre as características críticas de desempenho, é necessário considerar a aplicação do composto. Para carros esportivos, como os de competição, o melhor composto para a banda de rodagem seria o 15-45. Este composto apresenta maior aderência no piso molhado, dessa forma, responde melhor às frenagens, e ainda apresenta a menor dureza dentre os compostos analisados, o que torna o composto da banda de rodagem mais aderente ao piso.

Por outro lado, considerando-se aplicação de pneus em caminhões e ônibus, o principal requisito é que o pneu tenha um alto rendimento quilométrico, isto é, baixo desgaste abrasivo. Para atendimento dessa característica, o ideal seria a amostra 60-0, que apresentou o menor desgaste abrasivo. Contudo, essa mesma amostra apresenta o maior valor resistência ao rolamento, o que significa maior consumo de combustível (e consequente poluição atmosférica), embora, em baixas velocidades, a resistência ao rolamento é pouco importante. Por essas mesmas razões, para veículos de passeio o composto mais adequado também seria o 60-0. 


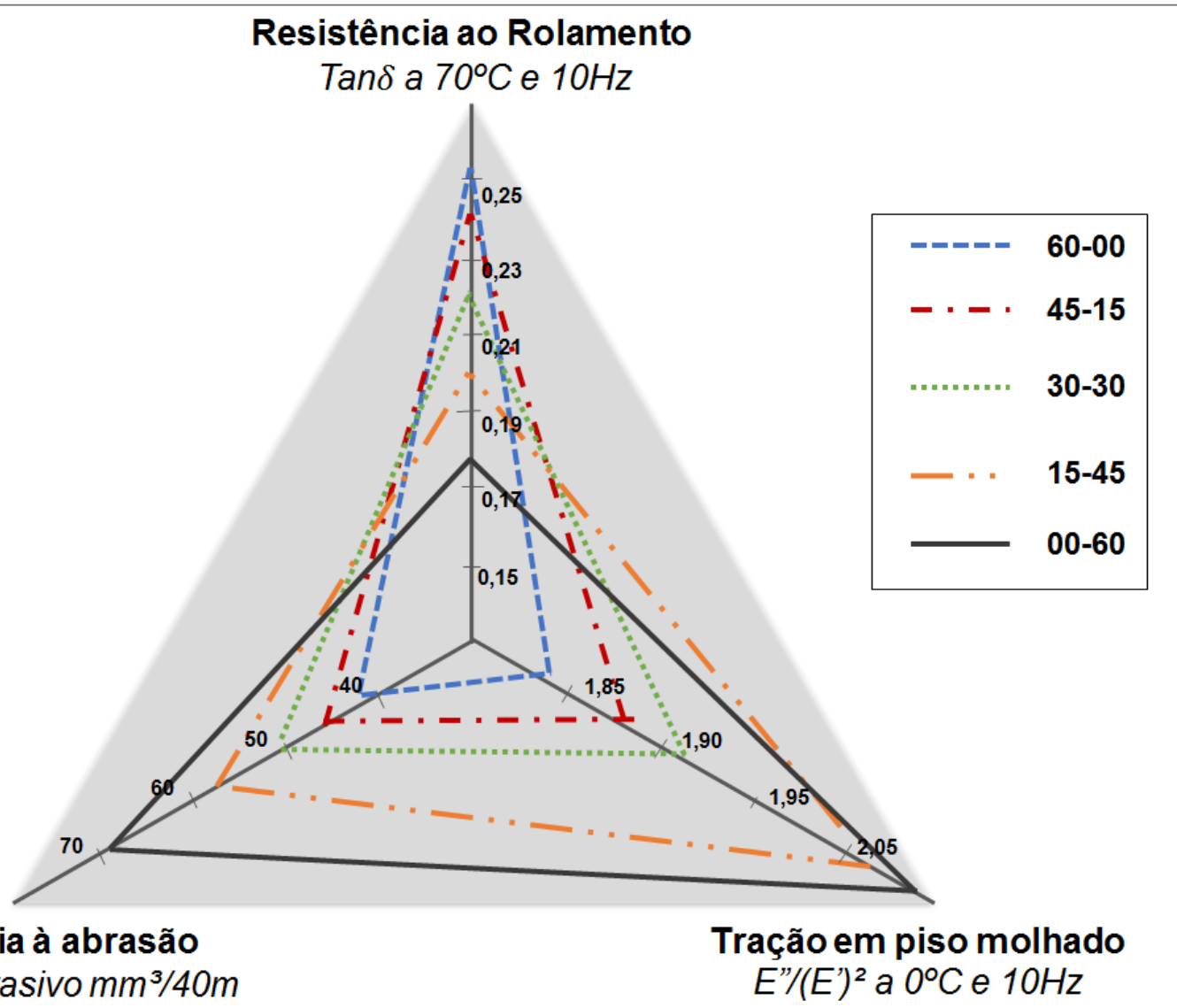

Resistência à abrasão

Desgaste abrasivo $\mathrm{mm}^{3} / 40 \mathrm{~m}$
$E^{\prime \prime} /\left(E^{\prime}\right)^{2}$ a $0^{\circ} \mathrm{C}$ e $10 \mathrm{~Hz}$

Figura 8. Formação do triângulo mágico a partir dos resultados de ensaio

\section{CONCLUSÕES}

Empregando-se os materiais e condições experimentais selecionados pôde-se concluir que:

- Os compostos com maior conteúdo de sílica apresentaram maior desgaste abrasivo, tornando assim o negro de fumo a melhor carga para otimização da resistência à abrasão.

- Os valores do fator de amortecimento tanס, obtidos a $0^{\circ} \mathrm{C}$ e $10 \mathrm{~Hz}$, aumentaram proporcionalmente com o aumento de sílica no composto, sendo esta carga mais adequada para otimização da característica de aderência ao piso molhado.

- Os valores do fator de amortecimento tan $\delta$, a $70^{\circ} \mathrm{C}$ e $10 \mathrm{~Hz}$ diminuíram com o aumento da quantidade de sílica no composto, o que torna esta carga também adequada à baixa resistência ao rolamento.

A interpretação de todos os resultados de ensaio obtidos nesse trabalho, cuja síntese está apresentada nas conclusões acima, permite a conclusão genérica de que a escolha do composto ideal, em relação as cargas estudadas, só pode ser feita considerando-se a aplicação especifica de um dado pneu. Em outras palavras, embora não exista um composto ótimo geral a ser indicado pelos resultados aqui obtidos, os dados resultantes desse trabalho, permitem que, a partir de uma dada aplicação e para um pneu específico, seja possível estabelecer critérios para a escolha do composto adequado. 


\section{Agradecimentos}

Os autores agradecem a Pirelli Pneus Ltda., Santo André - SP, nas pessoas dos Engos. Vinicius Lindo e Oswaldo Ortoni, pela inestimável colaboração ao longo da execução deste trabalho. Agradecem também a equipe do laboratório da engenharia de materiais pela assistência na realização dos ensaios.

\section{REFERÊNCIAS}

$1 \quad$ Brinke A. Silica reinforced tyre rubbers: mechanistic aspects of the role of coupling agents [thesis (Ph. D)]. Enschede: University of Twente; 2005.

2 Lindo VB. Obtenção, caracterização e aplicação na indústria pneumática, de sílicas precipitadas a partir de silicato de sódio [dissertação de mestrado]. Santo André: Universidade Federal do ABC; 2013, cap. 1 e 3.

3 Dierkes W. Economic mixing of silica-rubber compounds: interaction between the chemistry of the silica-silane reaction and the physics of mixing [thesis (Ph. D)]. Enschede: University of Twente; 2005.

4 Leblanc JL. Rubber-filler interactions and rheological properties in filled compounds. Progress in Polymer Science. 2002;27(4): 627-687.

5 Veiga VD. Influência da combinação sílica/negro de fumo e das etapas de processamento no desempenho de bandas de rodagem de pneu de carga [dissertação de mestrado]. Caxias do Sul: Universidade de Caxias do Sul; 2015.

6 Braum MV. Melhoria da interação polímero-carga através do uso de borracha de polibutadieno epoxidada [dissertação de mestrado]. Porto Alegre: Instituto de Química, Universidade Federal do Rio Grande do Sul; 2006.

7 Martins AF. et al. Propriedades mecânicas e dinâmico-mecânicas de composições de policloropreno com negro de fumo. Polímeros: Ciência e Tecnologia. 2002;12(3); 147152.

8 Lindo VB. Obtenção, caracterização e aplicação na indústria pneumática, de sílicas precipitadas a partir de silicato de sódio [dissertação de mestrado]. Santo André: Universidade Federal do ABC; 2013, cap. 4.

9 Medalia Al, Juencel RR, Collins JM. Carbon Black. In: developments in rubber technology: improving Product Performance. London: Applied Science; 1979. p. 151 181.

10 Wang M, Wolff S, Tan E. Filler-elastomer interactions. Part VIII. The role of the distance between filler aggregates in the dynamic properties of filler vulcanizates. Germany; 1992. 